American Journal of Animal and Veterinary Sciences 4 (4): 129-135, 2009

ISSN 1557-4555

(C) 2009 Science Publications

\title{
The Porcine Mitochondrial Transcription Factor a Gene: Molecular Characterization, Radiation Hybrid Mapping and Genetic Diversity among 12 Pig Breeds
}

\author{
${ }^{1,2}$ Tanja Kunej, ${ }^{1}$ Xiao-Lin Wu, ${ }^{1}$ Jennifer J. Michal, \\ ${ }^{2}$ Tamara Milosevic Berlic, ${ }^{1}$ Zhihua Jiang and ${ }^{2}$ Peter Dovc \\ ${ }^{1}$ Department of Animal Sciences, Washington State University, \\ Pullman, WA 99164-6351, USA \\ ${ }^{2}$ Department of Animal Science, University of Ljubljana, \\ Groblje 3, SI- 1230 Domzale, Slovenia
}

\begin{abstract}
Problem statement: Mitochondrial transcription factor A (TFAM) is a nucleus-encoded protein that is a key activator of mitochondrial transcription as well as a major participant in mitochondrial genome replication. Genomic characterization of the porcine TFAM gene is, therefore, necessary to determine its involvement in regulation of fat depots and meat quality traits in pigs. Approach: Genomic DNA sequence was determined using a comparative in silico annotation approach. RT-PCR was used for analysis of alternative splicing. Genome location was determined using Radiation Hybrid (RH) mapping. Genetic marker was identified by sequencing and genotyped by the PCR-RFLP method with SacI. GENEPOP version 3.3 software was used for statistic analysis. Results: We determined both full-length cDNA and genomic DNA sequences of the porcine TFAM gene. Gene expression analysis revealed an alternative 5' splice site, which excludes exon 4 of the pig gene. We assigned this gene to porcine chromosome 14 (SSC14). A G/A substitution was detected in intron 1 of porcine TFAM gene and genotyped on a total of 252 animals, including 165 from seven Chinese and 87 from five Western pig breeds. The Bayesian analysis via MCMC (Markov chain Monte Carlo) revealed that these two groups of pigs were well separated at this locus during the breed history; $95 \%$ of the posterior difference of TFAM allelic frequency between these two pig groups was greater than zero. Conclusion/Recommendations: All these data provided basic genomic information needed for further functional characterization of the porcine TFAM gene. Because marked differences in fat and lean tissue deposition exist between Western and Chinese pig breeds, the G557A mutation in the TFAM gene deserves further evaluation to determine its phenotypic effect on fattening and carcass traits in commercial pig populations.
\end{abstract}

Key words: alternative splicing, annotation, diversity, mapping, pig, TFAM

\section{INTRODUCTION}

Mitochondrial transcription Factor A (TFAM; previously known as mtTFA) is an integral part of the basal mitochondrial transcription machinery in mammals $^{[1]}$. It is required for transcription of mammalian mitochondrial DNA (mtDNA) from the light-strand and heavy-strand promoters in the presence of mitochondrial transcription factors B1 (TFB1M), B2 (TFB2M) and mitochondrial RNA polymerase $(\text { POLRMT })^{[2]}$. Transcription from the light-strand produces a short RNA primer, which is required for mtDNA replication. TFAM is also involved in the regulation of the mtDNA copy number ${ }^{[3]}$. For example, mice heterozygous for a targeted mutation of the Tfam gene exhibited reduced mtDNA copy number, while homozygous knockout embryos exhibited severe mtDNA loss and embryonic lethality, probably due to abolished transcription dependent priming of mtDNA replication ${ }^{[2,3]}$. In mammals, the TFAM gene has already been isolated and characterized in human ${ }^{[4]}$, mouse ${ }^{[5]}$, rat $^{[6]}$, cattle ${ }^{[7]}$ and silvered leaf-monkey ${ }^{[8]}$. The TFAM gene sequences for orangutan (CR857400), chimpanzee (XM_521481) and dog (XM_546107) were also deposited in the GenBank database (http://www.ncbi.nlm.nih.gov/). Here we report annotation, alternative splicing identification, potential miRNA target site detection, mapping and genetic diversity of porcine TFAM gene.

\section{Corresponding Author: Peter Dovc, Department of Animal Science, Biotechnical Faculty, University of Ljubljana,} Domzale, Slovenia Tel: +38617217836; Fax: +38617241005 


\section{MATERIALS AND METHODS}

\section{Molecular characterization of the porcine TFAM gene:}

Comparative annotation: The comparative approach for annotation of the porcine TFAM gene was described previously ${ }^{[7]}$. Basically, this annotation process consists of three steps. In step 1, we used the well-annotated human mRNA sequence as reference for BLAST searches to obtain the pig orthologous Expressed Sequence Tag (EST) sequences from the public database. Some of these ESTs were then selected for assembly and used for BLAST searches against the pig EST database for further extension of the cDNA sequence. In step 2, this extended cDNA sequence was further used as a query to retrieve genomic DNA sequence of the porcine TFAM gene and their alignment revealed its genomic organization. In step 3, the genomic DNA was used as a template against the EST databases for an electronic rapid amplification of cDNA ends (e-RACE) to generate a full-length cDNA sequence for the porcine gene. Such a process led to a comprehensive annotation of the porcine TFAM gene at both cDNA and genomic DNA levels.

Detection of alternative splicing forms: The total RNA was extracted by homogenization of $0.5 \mathrm{~g}$ of adipose and muscle tissues using TRIzol reagent according to the manufacturer's instructions (Gibco BRL, Gaithersburg, MD). The mRNA was then reverse-transcribed to generate cDNA (RT-Superscript, Gibco BRL, Gaithersburg, MD). One $\mu$ l of each cDNA mixture was amplified using the primers: 5'-CCG TTC AGT TTT GCG TAT GTA-3' (upstream primer; covering the border between the exons 1 and 2) and 5'GCT CAA CCT TCT ATT TCA ACA CT-3' (downstream primer; exon 7). PCR was performed for 30 cycles under the conditions of denaturation at $94^{\circ} \mathrm{C}$ for $45 \mathrm{sec}$, annealing at $57^{\circ} \mathrm{C}$ for $45 \mathrm{sec}$ and extension at $72^{\circ} \mathrm{C}$ for $1 \mathrm{~min}$ using the MJ Research PTC-200 DNA Engine Thermal Cycler (MJ Research, Waltham, MA). PCR product was electrophoresed through a $1 \%$ agarose gel and sequenced on ABI310 genetic anaylser (Applied Biosystems, Foster City, CA).

In silico miRNA binding site search: The search for predicted miRNA targets in the porcine TFAM gene was performed using TargetScan, Release 5.1 software. The predicted miRNA target site was compared with 3'-UTR sequences of the TFAM gene in several mammalian species, in armadillo and in platypus.

Radiation hybrid mapping of TFAM gene in the porcine genome:

Gene selection: A total of five genes, including TFAM, PCDH15, ZWINT, PHYHIPL, CDC2 were selected from the human genome resources provided by the National Center for Biotechnology Information (NCBI) at an average $\sim 1.7 \mathrm{Mb}$ interval (Table 1). These genes had orthologous sequence information available in pig, such as cDNA, genomic or EST sequences. Primer design was completed using the online oligonucleotide design tool Primer $3^{[9]}$. In order to avoid selecting homologous primers that might result in amplification of hamster DNA while screening the porcine/hamster panel DNA, each primer was compared to known mouse sequences for a particular gene using the NCBI BLAST tool. Primer specificity for the RH panel was verified using pure pig and hamster DNA in PCR amplifications. Primer pairs that amplified hamster DNA were omitted and redesigned.

RH panel typing: The RH panel source, marker typing procedure and RH map construction strategy were described previously ${ }^{[10]}$. In brief, DNA from a porcine/hamster RH panel was purchased from ResGen Invitrogen Corporation (Huntsville, AL, USA), which consisted of 94 cell lines, a porcine and a hamster DNA control. PCR reactions with a final volume of $10 \mu \mathrm{L}$ were performed under the conditions as follows: $95^{\circ} \mathrm{C}$ for $10 \mathrm{~min}, 32$ cycles of $94^{\circ} \mathrm{C}$ for $30 \mathrm{sec}, 56,57$ or $61^{\circ} \mathrm{C}$ (Table 1) for $30 \mathrm{sec}$ and $72^{\circ} \mathrm{C}$ for $30 \mathrm{sec}$, followed by a further $5 \mathrm{~min}$ extension at $72^{\circ} \mathrm{C}$. PCR products were then examined by electrophoresis on a $1.5 \%$ agarose gel in $1 \mathrm{X}$ TBE buffer. Gels were stained with ethidium bromide and photographed. Each gel was scored twice in order to eliminate possible scoring errors. The bands were designated into three classes: Positive (1), negative (0) and faint (?) for each gel image.

RH map construction: The RH maps were constructed using RHMAP (3.0), a statistical package for multipoint RH mapping developed by Boehnke et al. ${ }^{[11]}$. The RH2PT program was used to estimate the locusspecific retention probabilities and two-point LOD scores for linkage of the various marker pairs and to generate linkage groups with a LOD score of 4.0, 6.0 and 8.0. Framework maps were first established by RHMAPLIK at odds greater than 1000:1 (SAVMAX $=15$ and PRTMAX = 5) with a stepwise locus ordering strategy. The best comprehensive map was constructed with information from both RHMINBRK and RHMAXLIK analysis using the same ordering strategy at an equal retention model (SAVMAX = PRTMAX $=5$ ).

Genetic diversity of TFAM gene among 12 pig breeds:

Animals and DNA preparation: A total of 252 animals, including 165 from seven Chinese pig breeds and 87 from five Western pig breeds were used in this study. 
American J. Animal \& Vet. Sci., 4 (4): 129-135, 2009

Table 1: Primer sequences and PCR conditions for radiation hybrid mapping

\begin{tabular}{|c|c|c|c|c|c|c|}
\hline $\begin{array}{l}\text { Gene } \\
\text { symbol }\end{array}$ & Gene description & $\begin{array}{l}\text { HSA10 } \\
(\mathrm{Mb})\end{array}$ & $\begin{array}{l}\text { Forward primer }\left(5^{\prime} \rightarrow 3^{\prime}\right) \\
\text { Reverse primer }\left(5^{\prime \prime} \rightarrow 3^{\prime}\right)\end{array}$ & $\begin{array}{l}\text { Size } \\
(\mathrm{bp})\end{array}$ & $\begin{array}{l}\mathrm{Tm} \\
\left({ }^{\circ} \mathrm{C}\right) \\
\end{array}$ & Accession no. \\
\hline PCDH15 & Protocadherin 15 & 55.28 & $\begin{array}{l}\text { TTTTGGATGGCAAACTACTCG } \\
\text { GTAGCTGACCAAAACCACCAA }\end{array}$ & 212 & 56 & BP151124 \\
\hline ZWINT & ZW10 interactor & 57.78 & $\begin{array}{l}\text { AGAAACACCCAACAACTAGGGGGTA } \\
\text { AGCTTCCAGGTGTTTTCATGATTGA }\end{array}$ & 314 & 61 & CF363573 \\
\hline TFAM & $\begin{array}{l}\text { Mitochondrial transcription } \\
\text { factor A }\end{array}$ & 59.82 & $\begin{array}{l}\text { CTTGCTGAAGATGATAAAGTTCGTT } \\
\text { GCTCAACCTTCTATTTCAACACT }\end{array}$ & 150 & 61 & AY923074 \\
\hline PHYHIPL & $\begin{array}{l}\text { Phytanoyl-CoA hydroxylase } \\
\text { interacting protein-like }\end{array}$ & 60.61 & $\begin{array}{l}\text { AGAATTATCAGCACTTCCAACATG } \\
\text { AAGCAGACTAGATCAGAGGCAATTA }\end{array}$ & 362 & 57 & BQ599786 \\
\hline $\mathrm{CDC} 2$ & Cell divison cycle 2 protein & 62.21 & $\begin{array}{l}\text { ATGGCACTGAATCATCCATATTTT } \\
\text { GGTTGTTACAGTGGAATCTACAAACA }\end{array}$ & 261 & 56 & AJ660106 \\
\hline
\end{tabular}

Tm: Annealing temperature

Genomic DNA of the Chinese pig breeds was extracted at Nanjing Agricultural University from blood samples of Jinhua $(n=19)$, Neijiang $(n=28)$, Xian pig $(n=24)$, Ming pig $(\mathrm{n}=25)$, Wan $(\mathrm{n}=20)$, Jiangquhai $(\mathrm{n}=31)$ and Taoyuan $(n=18)$. As for Western pigs, skin tissue was sampled from five different pig breeds: Swedish Landrace $(\mathrm{n}=21)$, German Landrace $(\mathrm{n}=19)$, Piétrain $(\mathrm{n}=21)$, Yorkshire $(\mathrm{n}=18)$ and Krskopolje pig $(\mathrm{n}=8)$. These samples were collected from farms in Slovenia under the National Pig Improvement Program. Genomic DNA was prepared from skin tissue samples by standard phenol-chloroform-isoamyl alcohol (25:24:1) extraction.

Marker development: Primers from exon 1 and 2 were designed to amplify the sequence of entire intron 1: forward primer, 5'GTC ATT GTT GGG GTT GTT CTC T-3' and reverse primer: 5'-GTA TGA AGT CAT AGG CTT CTT TGGA-3'. A G557A substitution in intron 1 of the porcine TFAM gene (GenBank acc. no. AY923075) was detected by direct sequencing of PCR products on Chinese and Western DNA pools and then assayed on a total of 252 animals. The PCR reactions were performed under the same conditions as described above for RH panel genotyping. The PCR products were then digested with $2 \mathrm{U}$ of SacI (MBI Fermentas, Vilnius, Lithuania) at $37^{\circ} \mathrm{C}$ for $4 \mathrm{~h}$ and examined by electrophoresis on a $1.5 \%$ agarose gel with $1 \times \mathrm{TBE}$ buffer. The gels were stained with ethidium bromide and photographed.

Statistical analysis: GENEPOP version $3.3^{[12]}$ software was used to calculate allele frequency, observed $\left(\mathrm{H}_{\mathrm{O}}\right)$ and expected $\left(\mathrm{H}_{\mathrm{E}}\right)$ heterozygosity, Wright's $\mathrm{F}$ statistics at the TFAM locus in single pig populations and in pooled Chinese and Western pigs, respectively. Further, Bayesian Poisson modeling was used to reveal differentiation of TFAM gene frequency between Chinese and Western pigs by assuming that they are random samples from two distinct overall populations, respectively, using the following model: $\mathrm{x}_{\mathrm{ij}} \sim \operatorname{Poisson}\left(\theta_{\mathrm{i}} \mathrm{n}_{\mathrm{ij}}\right), \mathrm{i}=1,2 ; \mathrm{j}=1,2, . . \mathrm{n}_{\mathrm{i}}$

Where:

$\mathrm{x}_{\mathrm{ij}}$ and $\mathrm{n}_{\mathrm{ij}}=$ The number of allele $\mathrm{A}$ and the total number of alleles (A and $G$ ) in population $j$ from region $\mathrm{i}$ (i.e., $\mathrm{i}=1$ for Chinese pigs and $i=2$ for western pigs)

$\theta_{\mathrm{i}}=$ The Poisson mean for either region of pigs. conjugate gamma prior distribution is used for the allelic frequency parameter: $\theta_{\mathrm{i}} \sim \operatorname{Gamma}(\alpha, \beta)$

During the MCMC (Markov chain Monte Carlo) sampling, we ran a chain of 100,000 updates after a burn-in of 1,000 updates, with chain values saved at every 10th update. Thus, a total of 10,000 posterior samples were obtained to infer the distribution of allelic frequency parameter.

\section{RESULTS}

cDNA sequences compilation: The BLAST searches using the human TFAM cDNA (NM 003201) as a reference sequence retrieved fourteen porcine ESTs from the "ESTs others" database at NCBI that are orthologous to the human gene. Two ESTs (BW961355 + BP167625) were chosen for initial assembly, which was further extended by adding CV877499 to its 5' end and DB806415 to its 3' end. As such, these four ESTs formed a complete coding sequence of $1,798 \mathrm{bp}$ for the porcine TFAM gene, including $353 \mathrm{bp}$ of the 5'UTR region, $741 \mathrm{bp}$ of the coding sequence and $704 \mathrm{bp}$ of the 3'UTR region.

The coding sequence of $741 \mathrm{bp}$ was translated into a protein sequence of 246 amino acids for pig TFAM, the length being the same as in human, chimpanzee, cattle and dog, but two amino acids longer than in rat, three amino acids longer than in mouse and 38 amino acids shorter than in orangutan. The amino acid sequence showed $91 \%$ identity with cattle ${ }^{[7]}, 88 \%$ with 
dog (NW_139850), 71\% with human (M62810), chimpanzee (XM 521481) and orangutan (CR857400), $64 \%$ with rat (AJ312746), 62\% with mouse (U57939), $42 \%$ with frog (U35728), 40\% with chicken (AB059657), 22\% with fruit fly (AB045318) and 17\% with yeast (M73753), respectively.

Genomic organization: The cDNA sequence of 1,798 bp further retrieved a genomic DNA sequence of 171,235 bp (CU041308) from the GenBank database that contains the porcine TFAM gene. Alignment of both cDNA and genomic DNA sequence revealed that, like in other mammals, the pig gene has seven exons with six introns all completely sequenced. The size of each intron is: intron 1, $543 \mathrm{bp}$; intron 2, 2,052 bp; intron 3, $645 \mathrm{bp}$; intron 4, 4,587 bp; intron 5, 7,993 bp and intron $6,485 \mathrm{bp}$, respectively. The e-RACE further extended the 3'UTR from $704 \mathrm{bp}$ to $2,651 \mathrm{bp}$ with support of more than 50 ESTs. Therefore, the TFAM gene spans a region of at least 20,050 bp from transcriptional start site to transcriptional stop site in the porcine genome (CU041308).

Alternative splicing and miRNA target site: RT-PCR reactions amplified two amplicons, the predominant one with $660 \mathrm{bp}$ and another weaker amplicon with 558 bp. Sequencing analysis indicated that the latter amplicon does not contain a coding sequence of $102 \mathrm{bp}$ in exon 4 of the porcine gene (Fig. 1). The alternative splicing follows a "GT" site as a splicing donor site, which results in the shortening of exon 4 from $150 \mathrm{bp}$ to $48 \mathrm{bp}$. Therefore, the seven-exon structure remains preserved in the alternative splicing form of pig TFAM gene, but at the protein level it is 34 amino acids shorter compared to the long version.

The TargetScan search for conserved miRNA target sites revealed a highly conserved miR-27ab binding site in the 3'UTR region of the TFAM gene, which is conserved in nine species (Fig. 2). The sequence alignment revealed the conservation of the 3'UTR region in pig as well.

Chromosome assignment: Mapping of the porcine TFAM was performed by using a porcine/hamster RH panel, which consisted of 94 radiation hybrid cell lines plus pig and hamster DNA as controls. All five selected genes were successfully typed on the panel with a retention rate of $26.6 \%, 21.5 \%, 22.3 \%, 21.3 \%$ and $21.3 \%$ for CDC2, PHYHIPL, TFAM, ZWINT and PCDH15, respectively. The RH2PT program analysis assigned these five genes to one linkage group with a LOD score greater than 5 . As the porcine CDC2 gene has been previously placed on SSC14 by Nonneman and Rohrer ${ }^{[13]}$, any member of this linkage group is accordingly assigned to the same pig chromosome, including the porcine TFAM gene (Fig. 3). These five genes spanned a total of $129.5 \mathrm{cR}$ in distance.

Genetic diversity of TFAM gene among 12 pig breeds: A 792 bp fragment containing the first intron and a part of the first and the second exon of the porcine TFAM gene was amplified from DNA of 252 animals representing seven Chinese pig breeds and five Western pig breeds. The G557A substitution in the intron 1 (GenBank acc. no. AY923075) was identified using restriction enzyme SacI. The frequency of allele A was high (from 0.917-1.000) in the five Western pig breeds, whereas it was moderate (from $0.500-0.750$ ) in Chinese pig breeds except Jinhua and Taoyuan (Table 2).
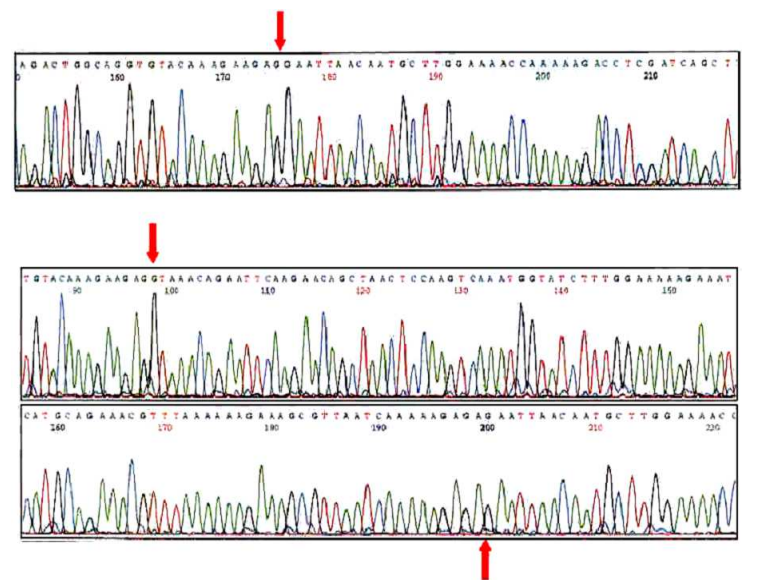

Fig. 1: Alternative splicing of the porcine TFAM gene. The upper panel shows the shorter splicing form lacking $102 \mathrm{bp}$ in comparison to the longer splicing form containing an additional 34 codons (lower panel)

$\ldots \ldots .290 \ldots \ldots 300 \ldots \ldots .310 \ldots . .3$ Bta --GAUAAAGGUAAAUCAGACUGUGAAG--UUUUUUUAUAC Mmu --GAU-GGGCUAGA-UAGACUGUGAAG-UGACUUUCACAC

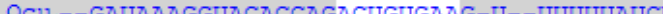
EeU --GGGAAAGGUAAACCAGACUGUGAAGUUUCUUUUUAUAC Cfa--GAUAAAGGUAAAUCAGACUGUGA.AG-UUUUUUUUAUAC Dno --GAUAAAGGUAA.AUCAGACUGUGA.AC-UUUUUUUU----

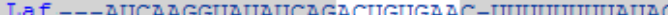
Mdo UAGAUAAGGAUAA.AUCA.AACUGUGA.AA-UAUUUUUGAUAC Oan --GAAAAAGUAAAAUUGAACUGUGA.AU-UUUUUUUUCC-$\operatorname{miR}-27 \mathrm{ab}$

Fig. 2: Highly conserved miRNA target site in the 3'UTR region of the TFAM gene identified using TargetScan software. The miR-27ab specific target region is highlighted. Bta: Bos taurus; Mmu: Mus musculus; Ocu: Oryctolagus cuniculus; Eeu: Erinaceus europaeus; Cfa: Canis familiaris; Dno: Dasypus novemcinctus; Laf: Loxodonta africana; Mdo: Monodelphis domestica; Oan: Ornithorhynchus anatinus 
American J. Animal \& Vet. Sci., 4 (4): 129-135, 2009

Table 2: Allele frequency, heterozygosity and Wright's F statistic $\left(F_{I S}\right)$ of TFAM gene in Chinese and Western pig populations ${ }^{1,2}$

\begin{tabular}{|c|c|c|c|c|c|c|c|c|c|c|}
\hline \multirow[b]{2}{*}{ Pig breed } & \multirow[b]{2}{*}{$\mathrm{N}$} & \multicolumn{3}{|c|}{ Genotypes } & \multicolumn{2}{|c|}{ Allele frequency } & \multicolumn{2}{|c|}{ Heterozygosity } & \multicolumn{2}{|l|}{ Fis } \\
\hline & & GG & GA & AA & G & A & $\mathrm{H}_{\mathrm{O}}$ & $\mathrm{H}_{\mathrm{E}}$ & $\mathrm{W}$ and $\mathrm{C}$ & $\mathrm{R}$ and $\mathrm{H}$ \\
\hline \multicolumn{11}{|c|}{ Chinese pig breeds } \\
\hline Neijiang & 28 & 2 & 17 & 9 & 0.375 & 0.625 & 0.607 & 0.477 & -0.279 & -0.288 \\
\hline Xian pig & 24 & 4 & 16 & 4 & 0.500 & 0.500 & 0.667 & 0.511 & -0.314 & -0.319 \\
\hline Ming pig & 25 & 7 & 9 & 9 & 0.460 & 0.540 & 0.360 & 0.507 & 0.294 & 0.302 \\
\hline Wan pig & 20 & 0 & 10 & 10 & 0.250 & 0.750 & 0.500 & 0.385 & -0.310 & -0.316 \\
\hline Jiangquhai & 31 & 8 & 11 & 12 & 0.436 & 0.565 & 0.355 & 0.500 & 0.293 & 0.300 \\
\hline Taoyuan & 18 & 1 & 0 & 17 & 0.056 & 0.944 & 0.000 & 0.109 & 1.000 & 1.059 \\
\hline Jinhua & 19 & 0 & 0 & 19 & 0.000 & 1.000 & - & - & - & - \\
\hline Chinese & 165 & 22 & 63 & 80 & 0.324 & 0.676 & 0.382 & 0.440 & 0.132 & 0.132 \\
\hline \multicolumn{11}{|c|}{ European pig breeds } \\
\hline Swedish & 21 & 0 & 1 & 20 & 0.024 & 0.976 & 0.048 & 0.048 & 0.000 & 0.000 \\
\hline \multicolumn{11}{|l|}{ Landrace } \\
\hline Piétrain & 21 & 0 & 0 & 21 & 0.000 & 1.000 & & & - & - \\
\hline Yorkshire & 18 & 0 & 3 & 15 & 0.083 & 0.917 & 0.167 & 0.157 & -0.063 & -0.064 \\
\hline Krskopolje pig & 8 & 0 & 0 & 8 & 0.000 & 1.000 & - & - & - & - \\
\hline German & 19 & 0 & 0 & 19 & 0.000 & 1.000 & - & - & - & - \\
\hline \multicolumn{11}{|l|}{ Landrace } \\
\hline Western & 87 & 0 & 4 & 83 & 0.023 & 0.977 & 0.046 & 0.045 & -0.017 & -0.017 \\
\hline
\end{tabular}

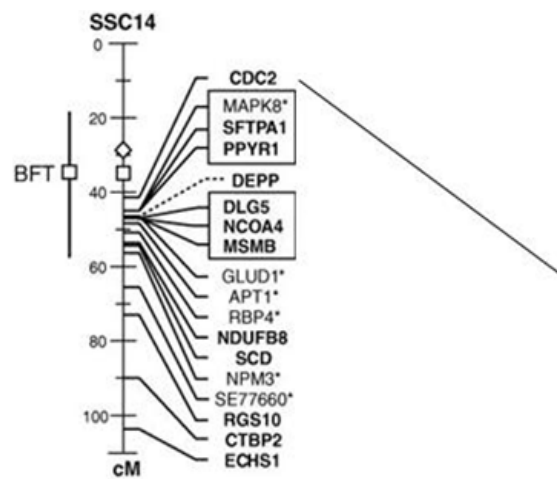

(a)

Fig. 3: A map of the SSC $14 q$ published by ${ }^{[14]}$ (a) and a partial pig RH map of the SSC 14q from this study (b). QTL position for Backfat (BFT) is shown as a bar on the left with the peak indicated with a square symbol on the bar and on the linkage map. Distances are in centimorgans $(\mathrm{cM})$ and centirays $(\mathrm{cR})$

The high frequency of allele A in two Chinese breeds, Jinghua and Taoyuan, could be due to introduction of Western pig blood during the breeding history of the breed. The Hardy-Weinberg test indicated that none of these populations exhibited an excess of heterozygotes $(\mathrm{P}=0.14 \sim 1.00)$. However, Taoyuan pigs showed significant heterozygote deficit $(\mathrm{P}=0.0286)$ while all the other pig populations did not $(\mathrm{P}=0.10 \sim 0.98)$. On average, both $\mathrm{H}_{\mathrm{O}}$ and $\mathrm{H}_{\mathrm{E}}$ were higher in Chinese breeds $(0.382$ and 0.440 , respectively) than in western breeds

(0.046 and 0.045 , respectively). Using Bayesian Poisson modeling strategy, the frequency (posterior mean \pm standard deviation) of allele $A$ of the TFAM gene was estimated to be $0.677 \pm 0.045$ for Chinese pig breeds and $0.967 \pm 0.747$ for Western pig breeds, respectively. The corresponding $95 \%$ quartile of the posterior mean was from $0.591-0.768$ for Chinese pigs and from $0.858-1.000$ for Western pigs.

\section{DISCUSSION}

In the present study, we developed a simple, but reliable procedure to characterize the genomic features of porcine TFAM gene. First, we used a comparative approach to retrieve cDNA sequence of the porcine gene from the EST database: four ESTs (BW961355, BP167625, CV877499 and DB806415) contributed to the formation of a full-length cDNA sequence. Second, this full-length cDNA sequence was then used to search for its genomic DNA sequence against the pig genome sequencing database, which revealed that the pig gene contains seven exons. Third, mRNA extracted from two tissues was used to examine whether this gene has alternative splicing forms. Two splicing forms were confirmed by sequencing. Last, bioinformatics search revealed a miR-27 target site in the 3'UTR that was conserved in ten species including pig. Interestingly, the role of miR-27 in adipogenesis has been reported just recently ${ }^{[14]}$. However, the experimental validation of the predicted miR-27ab target in the porcine TFAM 3'UTR region as well as in other species needs to be performed in the future. 
RH mapping assigned the TFAM and four neighbor genes on SSC14. The gene order for PCDH15, ZWINT, TFAM, PHYHIPL and CDC2 was conserved in both human and pig genomes, but the orientation of the gene cluster in both species needs to be investigated by further mapping efforts. Interestingly, this linkage group of five genes falls into a region where the QTL for backfat thickness has been reported by Rohrer and Keele $^{[15,16]}$. Recently, Jiang and colleagues ${ }^{[7]}$ have reported a significant association of TFAM promoter polymorphisms with marbling scores and subcutaneous fat depth in Wagyu $x$ Limousin cattle $F_{2}$ crosses. This may imply that the porcine TFAM gene may be a strong candidate gene for this backfat QTL on SSC14, indicating that the TFAM gene plays an important role in lipid metabolism, fat deposition in muscle and obesity in mammals in general.

Observed $\left(\mathrm{H}_{\mathrm{O}}\right)$ and expected heterozygosity $\left(\mathrm{H}_{\mathrm{E}}\right)$ was not dramatically different in these pig populations except for Taoyuan pigs, indicating no significant departure from Hardy-Weinberg equilibrium. This observation is in agreement with the calculated $F_{I S}$ statistic at this locus, which was low in most breeds except in Taoyuan pigs. The $\mathrm{F}_{\text {IS }}$ statistic measures the reduction in heterozygosity in an individual due to nonrandom mating within a population. Consistently, Hardy-Weinberg test indicates significant heterozygote deficit in Taoyuan pigs. This might be due to so-called Walhund effect, which is the result of the presence of subpopulations in the samples representing this breed. However, given that only 18 Taoyuan pigs were sampled, the significant heterozygote deficit could be due to sampling error as well. In addition, we noted that both $\mathrm{H}_{\mathrm{O}}$ and $\mathrm{H}_{\mathrm{E}}$ were higher in Chinese breeds than in Western breeds, which is similar to some previous reports ${ }^{[17]}$. As heterozygosity is a measure of genetic diversity, this result indicates that Chinese breeds exhibited higher within-population variation than Western pigs. Bayesian estimation indicated a clear allele differentiation between Chinese and Western pigs at this locus. Differentiation of allele frequency of the TFAM gene could reflect the fact that genes involved in energy metabolism were affected by different selection strategies in both groups of pig breeds.

\section{CONCLUSION}

The present study represents the first comprehensive investigation of the pig TFAM gene for its genomics features, such as cDNA sequence, genomic organization, alternative splicing and miRNA target site. We placed this gene to Sus scrofa chromosome 14 and developed a single nucleotide polymorphism in its intron 1. Because marked differences in fat and lean tissue deposition exist between Western and Chinese pig breeds, the mutation G557A in the TFAM gene deserves further evaluation to determine its phenotypic effect on fattening and carcass traits in commercial pig populations.

\section{ACKNOWLEDGEMENT}

This study was supported by the bilateral scientific exchange program between USA and Slovenia (project number: BI-US/03-04/17).

\section{REFERENCES}

1. Gaspari, M., M. Falkenberg., N.G. Larsson and C.M. Gustafsson, 2004. The mitochondrial RNA polymerase contributes critically to promoter specificity in mammalian cells. EMBO J., 23: 4606-4614. PMID: 15526033

2. Falkenberg, M., M. Gaspari, A. Rantanen, A. Trifunovic, N.G. Larsson and C.M. Gustafsson, 2002. Mitochondrial transcription factors B1 and B2 activate transcription of human mtDNA. Nat. Genet., 31: 289-294. PMID: 12068295

3. Larsson, N.G., J. Wang, H. Wilhelmsson and A. Oldfors et al., 1998. Mitochondrial transcription factor A is necessary for mtDNA maintenance and embryogenesis in mice. Nat. Genet., 18: 231-236. PMID: 9500544

4. Fisher, R.P. and D.A. Clayton, 1988. Purification and characterization of human mitochondrial transcription factor 1. Mol. Cell Biol., 8: 3496-509. PMID: 3211148.

5. Larsson, N.G., J.D. Garman, A. Oldfors, G.S. Barsh and D.A. Clayton, 1996. A single mouse gene encodes the mitochondrial transcription factor a and a testis-specific nuclear HMG-box protein. Nat. Genet., 13: 296-302. PMID: 8673128

6. Gadaleta, G., D. D'Elia, L. Capaccio, C. Saccone and G. Pepe, 1996. Isolation of a $25-\mathrm{kDa}$ protein binding to a curved DNA upstream the origin of the $\mathrm{L}$ strand replication in the rat mitochondrial genome. J. Biol. Chem., 271: 13537-13541. PMID: 8662779

7. Jiang, Z., T. Kunej, J.J. Michal, C.T Gaskins and J.J. Reeves et al., 2005. Significant associations of the mitochondrial transcription factor A promoter polymorphisms with marbling and subcutaneous fat depth in Wagyu $\times$ Limousin $\mathrm{F}_{2}$ crosses. Biochem. Biophys. Res. Commun., 334: 516-523. PMID: 16005429 
8. D'Errico, I., A. Reyes, M.M. Dinardo and G. Gadaleta, 2005. Study of the mitochondrial transcription factor A (Tfam) gene in the primate Presbytis cristata. Gene, 354: 117-124. PMID: 15978747

9. Rozen, S. and H.J. Skaletsky, 2000. Primer3 on the WWW for General Users and for Biologist Programmers. In: Bioinformatics Methods and Protocols: Methods in Molecular Biology, Krawetz, S. and S. Misener (Eds.). Humana Press, Totowa, NJ., pp: 365-386.

10. Jiang, Z., H. He, N. Hamasima, H. Suzuki and G. Verrinder, 2002. Comparative mapping of Homo sapiens chromosome 4 (HSA4) and Sus scrofa chromosome 8 (SSC8) using orthologous genes representing different cytogenetic bands as landmarks. Genome, 45: 147-156. PMID: 11908657

11. Boehnke, M., K. Lange and D.R. Cox, 2001. Statistical methods for multipoint radiation hybrid mapping. Am. J. Hum. Genet., 49: 1174-1188. PMID: 1746551

12. Raymond, M. and F. Rousset, 1995. GENEPOP (Version 3.3): Population genetics software for exact tests and ecumenicism. J. Hered., 86: 248-249. http://jhered.oxfordjournals.org/cgi/reprint/86/3/24 8

13. Lin, Q., Z. Gao, R.M. Alarcon, J. Ye and Z. Yun, 2009. A role of miR-27 in the regulation of adipogenesis. FEBS J., 276: 2348-2358. PMID: 19348006

14. Nonneman, D. and G.A. Rohrer, 2004. Comparative mapping of human chromosome 10 to pig chromosomes 10 and 14. Anim. Genet., 35: 338-343. PMID: 15265077

15. Rohrer, G.A. and J.W. Keele, 1998a. Identification of quantitative trait loci affecting carcass composition in swine: I. Fat deposition traits. J. Anim. Sci., 76: 2247-2254. PMID: 9781479
16. Rohrer, G.A. and J.W. Keele, 1998b. Identification of quantitative trait loci affecting carcass composition in swine: II. Muscling and wholesale product yield traits. J. Anim. Sci., 76: 2255-2262. PMID: 9781480

17. Laval, G., N. Iannuccelli, C. Legault, D. Milan and M.A. Groenen et al., 2000. Genetic diversity of eleven European pig breeds. Genet. Sel. Evol., 32: 187-203. PMID: 14736401

18. Weir, B.S. and C.C. Cockerham, 1984. Estimating F-statistics for the analysis of population structure. Evolution, 38: 1358-1370. http://www.jstor.org/pss/2408641

19. Robertson, A. and W.G. Hill, 1984. Deviations from Hardy-Weinberg proportions: sampling variances and use in estimation of inbreeding coefficients. Genetics, 107:703-718. PMID: 6745643 